\title{
DISTANCE HISTOGRAM TO CENTROID AS A UNIQUE FEATURE TO RECOGNIZE OBJECTS
}

\author{
Pilar Arques, Rafael Molina, Mar Pujol, Ramon Rizo \\ Group: "Informatica Industrial e Inteligencia Artificial" \\ Universidad de Alicante \\ arques@.dccia.ua.es,rmolina@dccia.ua.es,mar@dccia.ua.es,rizo@dccia.ua.es
}

\begin{abstract}
Keywords: Feature extraction, Pattern Recognition, Histogram, Centroid.
Abstract: The shape of objects plays an essential role among the different aspects of visual information. A 2D silhouette often conveys enough information to allow the correct recognition of the original 3D object. Distance Histogram to Centroid will be used as the unique feature to totally describe an object and to distinguish it from all the other objects in the scene. The proposed system has been proved to be robust to discriminate between classes in a given set of objects The main advantages are the elimination of the feature selection process and avoiding the problem of dimensionality.
\end{abstract}

\section{INTRODUCTION}

The shape of objects plays an essential role among the different aspects of visual information (Marr, 1982). Therefore, it is a very powerful feature when used in similarity search and retrieval. Unlike colour and texture features, the shape of an object is strongly tied to the object functionality or identity. Unfortunately, semantically meaningful shapes are not easy to obtain from images, due to the poor performance of todays' automatic segmentation algorithms. Moreover, noise, occlusion and distortions introduced during the image formation process, make object extraction and similarity estimation a difficult task. Image formation is basically a projection of the $3 \mathrm{D}$ world onto the $2 \mathrm{D}$ space. A 2D silhouette often conveys enough information to allow the correct recognition of the original $3 \mathrm{D}$ object.

A new method to characterize objects is presented. It is based on the distance between the object centroid and all its contour points. A frequency histogram which characterizes the object is obtained from the distance.

The paper is organized as follows. In section 2, there is a brief description about desiderable properties for a shape representation method, and a short shape representation methods review. Section 3 is focused on the definition of the distance histogram to centroid. Section 4 shows several proves and examples of classification with our method. And, section 5 explains the conclusions and future work.

\section{FEATURES USED IN IMAGE INTERPRETATION}

To find or recognize an object in a scene it is necessary to extract its properties (colour, size, elongation ...) and keep them in a vector, namely feature vector. These feature vectors mathematically define and represent an object. Any process which transforms pixels in a more compact format of a superior level is a representation of an object. For example, in a first approximation the border of an object is defined by all the pixels in the frontier. A more efficient representation of the border is polygonal adjustment of contour points. Sometimes the pixels are not transformed; they form a representation which defines the object.

Evaluating the quality of the shape description method is a common problem in shape description research. Each application domain requires a suitable approximation. The chosen method depends on the features used to describe the objects shape and the application domain. Sometimes, the presence of noise in the image determines the description method. There is not a definitive method to evaluate the goodness of a shape description approximation, although shape representation schemes must have some desirable properties (Pitas, 2000):

- Uniqueness. It has a crucial importance in object recognition, because each object must have a unique representation. 
- Completeness. This refers to unambiguous representations. Invariance under geometrical transformations.

- Invariance under translation, rotation, scaling and reflection is very important for object recognition applications.

- Sensitivity. This is the ability of a representation scheme to reflect easily the differences between similar objects.

- Abstraction from detail. This refers to the ability of a representation to represent the basic features of a shape and to abstract from detail. This property is directly related to the robustness of the representation under noise conditions.

Shape representation techniques are generally characterized as being boundary-based or region-based. The former represents the shape by its outline, while the latter considers the shape as being composed of a set of two-dimensional regions. Techniques in both categories can be further subdivided into subcategories (Fontoura and Marcondes, 2001):

- Contour-based approaches: Spatial domain techniques (Parametric contours, Set of contour points, ...) and Transform domain techniques (Fourier transform of the parametric representation).

- Region-based approaches: Spatial domain techniques (Region Decomposition, Boundary Regions, ...) and Transform Domain Techniques (such as Gabor filters).

Selecting a set of features from the shape representation to characterize an object for a certain application is not easy, since the variability of the shapes and the specific characteristics of the application domain must be taken into consideration. Feature comparison can be understood as a way of quantifying the similiarity/dissimilarity between corresponding objects. This is a very difficult problem since it tries to mimic the human perception of similarity between objects (Papathomas, 1996).

\section{DISTANCE HISTOGRAM TO CENTROID}

Distance Histogram to Centroid will be used as the unique feature to totally describe an object and to distinguish it from all the other objects in the scene. The process of calculation can be dramatically speeded up using a unique property to characterize an object. The problem of dimensionality would be also avoided. The selection of an adequate classifier would even allow the system to recognize the shapes in real time.

For each region in the scene, the centroid (Figure 1 A) and the distance from it to every perimeter point
(Figure $1 \mathrm{~B}$ ) are calculated. The categories of the histogram are defined by the user. Once the Euclidean distance from the border points to the centroid is calculated, it is assigned to the correct category bear in mind the maximum distance from the border to the centroid. So the obtained histogram is invariable to rotation and to translation but not to scaling. To achieve scaling invariance the histogram must be normalize by dividing each histogram component by the object perimeter.
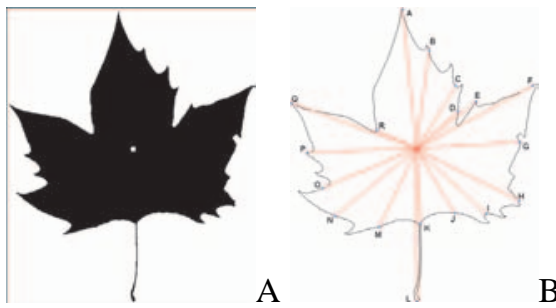

Figure 1: Centroid.

The Distance Histogram to Centroid is defined as follows:

Let $R$ be one region in the scene.

Let $P$ be the perimeter of region $R$.

Let $x=\left(\bar{x}_{R}, \bar{y}_{R}\right)$ be the centroid of region $R$.

Let $F_{1}, \cdots, F_{n}$ be the set of all the border points of region $R$.

The Euclidean distance between each border point and the centroid should be calculated by 1

$$
\operatorname{distance}\left(F_{i}\right)=\sqrt{\left(F_{i}(x)-\bar{x}_{R}\right)^{2}+\left(F_{i}(y)-\bar{y}_{R}\right)^{2}}
$$

Let $D_{\max }$ be the maximum distance from any border point to the centroid in region $R$.

Let $N C A T$ be the total number of categories in which the histogram is divided.

The equation 2 calculates the category for this distance.

$$
\operatorname{cat}\left(\operatorname{distance}\left(F_{i}\right)\right)=\operatorname{distance}\left(F_{i}\right) * \frac{N C A T}{D_{\max }}
$$

Let $H$ be the resulting histogram and let $H[1] . . H[N C A T]$ be the different resulting values for each category.

The proposed algorithm is as follows:

$$
\begin{aligned}
& \text { For every } F_{j} \text { do } \\
& \text { Calculate distance }\left(F_{j}\right) \\
& \text { Calculate } d=\operatorname{cat}\left(\operatorname{distance}\left(F_{j}\right)\right) \\
& H[d]++ \\
& \text { For } i=1 \text { to } i=N C A T \text { do } \\
& H[i]=H[i] / P
\end{aligned}
$$

Figure 2 shows an object with several rotation, translation and scaling transformations. The first obtained histogram (figure $3 \mathrm{~A}$ ) it is not invariable to scaling. 


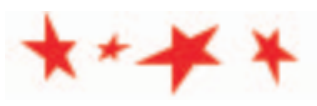

Figure 2: An object.
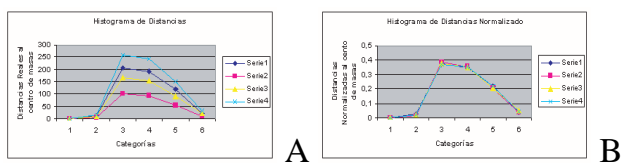

Figure 3: Histograms of figure 2.

To achieve scaling invariability normalization is applied (see figure $3 \mathrm{~B}$ ):

$$
\forall H_{i} \in H H_{i}=\frac{H_{i}}{P}
$$

\subsection{Example}

Table 1 introduces the coordinates and the centroid distance for the border points labelled A..R in figure $1 \mathrm{~B}$.

Table 1: Histogram Centroid Distance data useful.

\begin{tabular}{|c|c|c|}
\hline & Coordinates & Centroid distances \\
\hline A & $(225,1)$ & 279 \\
B & $(279,86)$ & 194 \\
C & $(336,157)$ & 146 \\
D & $(337,207)$ & 109 \\
E & $(375,186)$ & 152 \\
F & $(492,163)$ & 264 \\
G & $(465,269)$ & 211 \\
H & $(462,380)$ & 231 \\
I & $(395,408)$ & 191 \\
J & $(334,407)$ & 151 \\
K & $(262,427)$ & 149 \\
L & $(254,584)$ & 305 \\
M & $(175,435)$ & 175 \\
N & $(94,414)$ & 210 \\
O & $(65,356)$ & 204 \\
P & $(31,287)$ & 223 \\
Q & $(1,188)$ & 269 \\
R & $(172,246)$ & 88 \\
\hline
\end{tabular}

Table 2 shows de perimeter, centroid coordinates, maximum and minimum centroid distance to calculate the Distance Histogram to Centroid. In this case, just some border points are randomly chosen, but all the border points are actually needed to calculate the correct histogram.

Once all the data of the perimeter have been calculated, we must obtain its related histogram.

Let us suppose that the categories number in which we want to divide the histogram is 5 , as the maximum
Table 2: Global data from figure $1 \mathrm{~B}$.

\begin{tabular}{|c|c|}
\hline Coord. Centroid & $(254,278)$ \\
\hline Maximum Distance & 305 \\
\hline Minimum Distancie & 88 \\
\hline Perimeter & 2339 \\
\hline
\end{tabular}

distance is 305 , each category groups the distances in 5 different intervals with value 61 . For each category, the table 3 shows the distance range and the frequency for the labelled points in figure $1 \mathrm{~B}$.

Table 3: Distance Frequency Histogram from points in figure $1 \mathrm{~B}$.

\begin{tabular}{|c|c|c|c|c|c|}
\hline Category & 1 & 2 & 3 & 4 & 5 \\
\hline Interval & $0-60$ & $61-121$ & $122-182$ & $183-243$ & $244-305$ \\
\hline Frequency & 0 & 2 & 5 & 7 & 4 \\
\hline
\end{tabular}

The frequency histogram obtained in table 3 is rotation and/or translation invariable, because all the contour points are taken into consideration to calculate it, and the initial contour point has no influence in this histogram. However, this first histogram is totally conditionated by the object size. Just suppose a large object: it would have higher frequency values than the same object with a smaller size. To achieve the scaling invariability the frequency data are normalized by dividing it by the object perimeter value.

\section{EXPERIMENTS}

To verify that the Distance Histogram to Centroid defines a unique, robust, complete and invariable feature, real image proofs with different categories are been made.

Two kinds of real images have been selected: images from traffic signals (figures 4 and 6) and from library books (figure 10). To make the data base, the object is extracted from the scene (figures 5, 7 and 11 ), and the histogram distance to centroid is calculated (figures 8, 9 and 12).

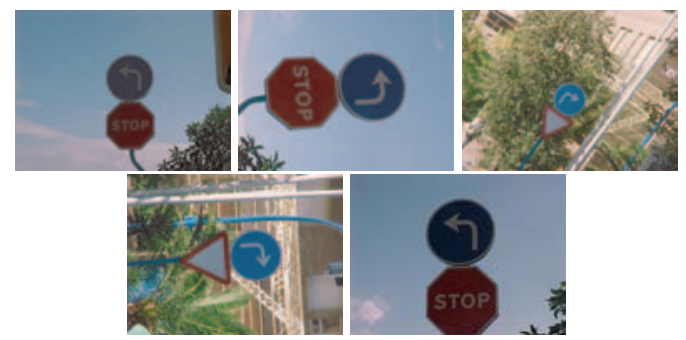

Figure 4: Traffic Signal. Original Image. 


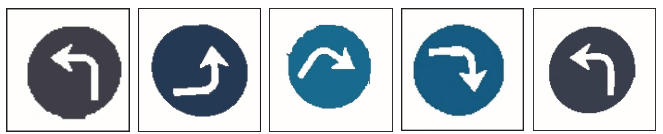

Figure 5: Traffic Signal 1. Extraction.

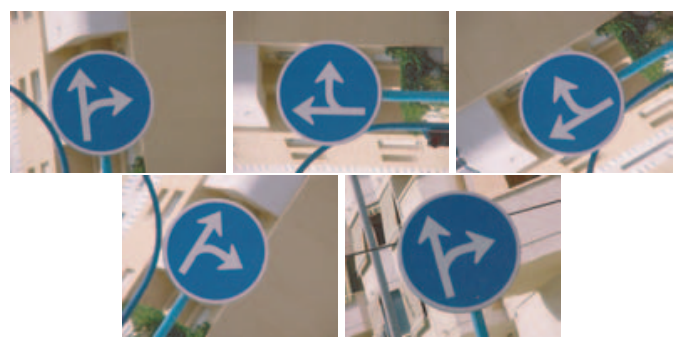

Figure 6: Traffic Signal. Original Image.

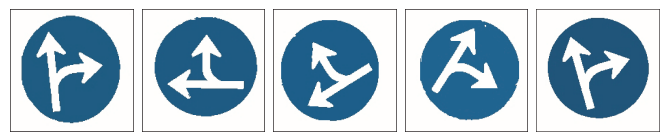

Figure 7: Traffic Signal 2. Extraction.

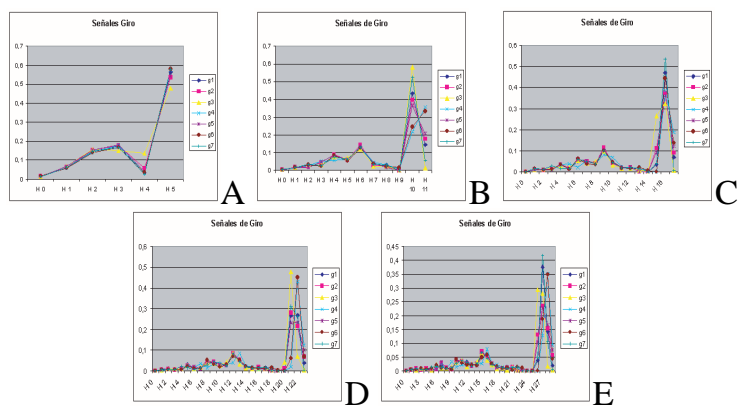

Figure 8: Traffic Signal 1. Histogram.

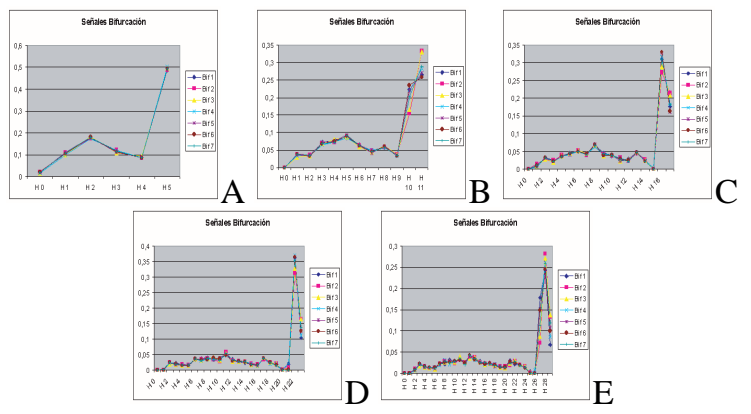

Figure 9: Traffic Signal 2. Histogram.

Histograms belonging to the same object have been grouped in the same figure. Figure 8 shows the histograms of traffic signal 1 (Figure 5) and figure 9 shows the histograms of traffic signal 2 (Figures 7).
Histograms have also been grouped by categories, that is, figure $8 \mathrm{~A}$ and figure $9 \mathrm{~A}$ represents 6 categories, figure $8 \mathrm{~B}$ and figure $9 \mathrm{~B}$ represents 12 categories, and so on. So depending on the type of the object in the database the number of categories for a correct discrimination is studied.

To verify if the Distance Histogram to Centroid characterizes univocally an object, we used another kind of images, the digits in a library book.

In figure 10 there are several images with different library books. Figure 11 shows the segmentation obtained applying (Arques et al., 2005) algorithm. Taking this segmentation as a starting point, each segmented region distance histogram to centroid is calculated.

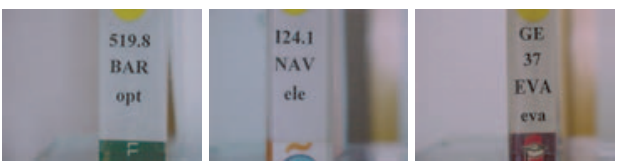

Figure 10: Library books. Original Image.

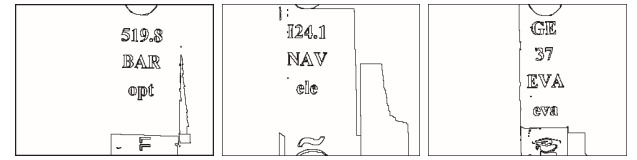

Figure 11: Library books. Segmented Image.

Figure 12 displays the Distance Histogram to Centroid for the library books digits, in this case we work with 18 categories, we used 20 samples of each digit. The graphical results show a different graphic for each digit except for 6 and 9 digits which have the same shape. So this method could be useful to recognize objects.

\subsection{Classification}

The task of a classifier in a full system is to use the feature vector provided by the feature extractor to assign the object to a category. Due to the fact that perfect classification performance is often impossible, a more general task is to determine the probability for each of the possible categories. The abstraction provided by the feature-vector representation of the input data enables the development of a largely domain-independence theory of classification (Duda et al., 2001).

The degree of difficulty of the classification problem depends on the variability in the feature values for objects in the same category relative to the difference between feature values for objects in different categories. 
Given a classification task of $M$ classes, $w_{1}, w_{2}$, ..., $w_{m}$ and an unknown pattern, which is represented by a feature vector $x$, we form the $\mathrm{M}$ conditional probabilities $P\left(w_{i} \mid x\right), i=1,2, \cdots, M$. Sometimes, these are also referred to as "a posteriori" probabilities. Each of them represents the probability of the unknown pattern to belong to the respective class $w_{i}$, given that the corresponding feature vector takes the value $x$. Let $\mu_{i}$ the average histogram of class $i$.

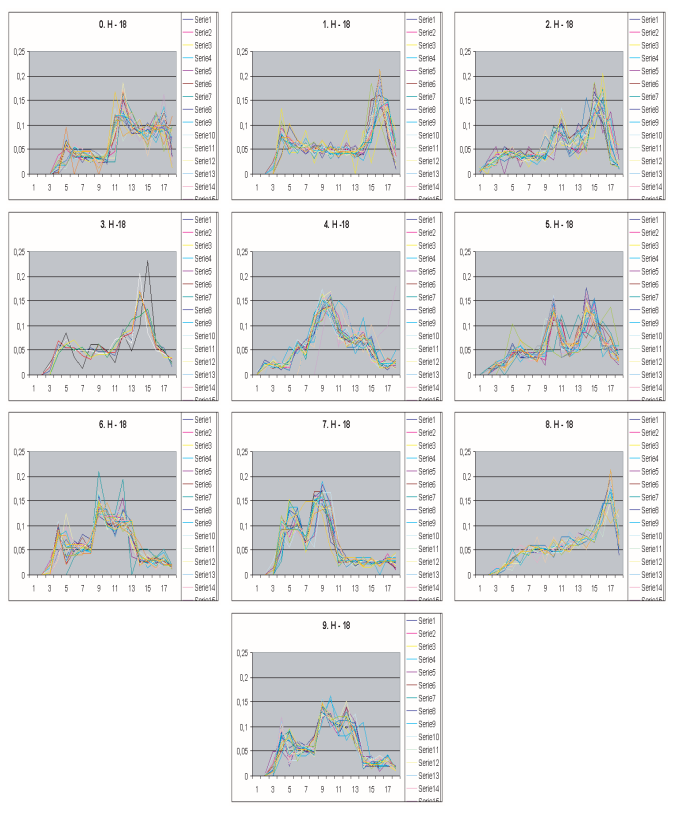

Figure 12: Library books. Histogram.

A method to verify the distance histogram to centroid as a set of features to classify objects is presented. 10 different classes have been defined, one for each digit, and the mean of each class is calculated. Then, different discriminant functions to classify are used:

\section{Euclidean Distance:}

$$
d_{\in}=\left\|x-\mu_{i}\right\|
$$

Thus, the feature vectors are assigned to classes according to their Euclidean distance from the respective mean points.

Figure 13 shows the graphic results obtained using Euclidean Distance as a discriminant function between classes. Each graphic represents the Euclidean Distance between the mean value of each class and all samples in database. The lowest value corresponds to samples which belong to $\mu_{i}$ class. In this case we have been used 12 categories.

\section{Kullback-Leibler Distance:}

The relative entropy or Kullback-Leibler distance (which is closely related to cross entropy, information divergence and information for discrimination) is

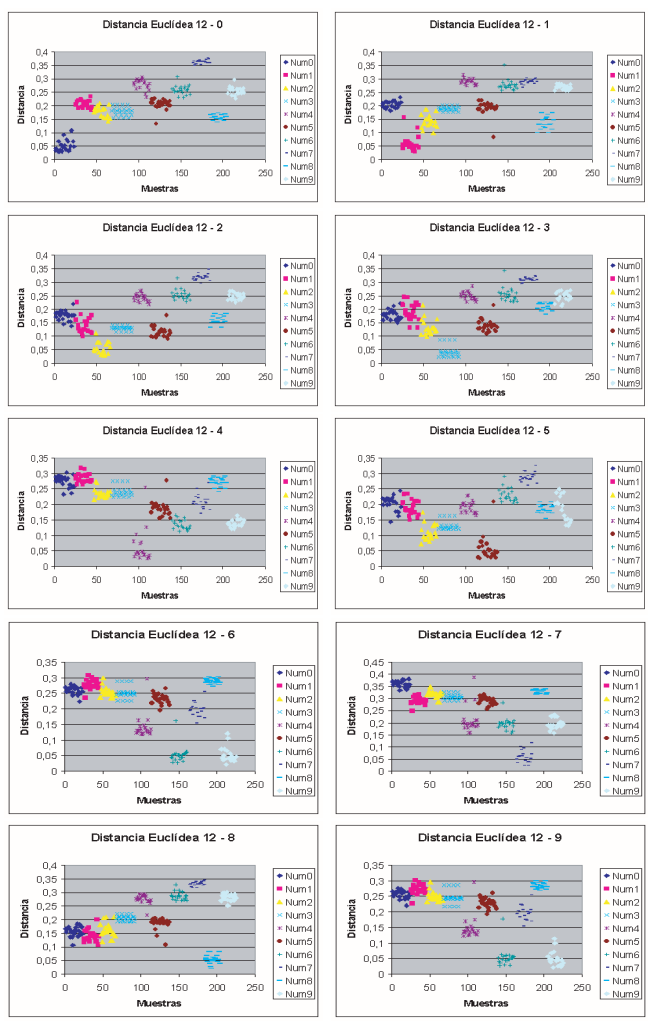

Figure 13: Euclidean Distance.

a measure of the distance between two distributions. For a multiclass problem, the divergence (KullbackLeibler distance) is computed for every class pair $w_{i}$, $w_{j}$. (Theodoridis and Koutroumbas, 1999)

$$
d_{K L}=\sum_{i=1}^{N} x_{i} \log \frac{x_{i}}{\mu_{i}}+\sum_{i=1}^{N} \mu_{i} \log \frac{\mu_{i}}{x_{i}}
$$

Figure 14 shows the graphic results obtained using Kullback-Leibler Distance as a discriminant function between classes. Each graphic represents KullbackLeibler Distance between the mean value of each class and all samples in database. The lowest value corresponds to samples which belong to $\mu_{i}$ class. In this case we have been used 18 categories.

In both cases, Euclidean Distance and KullbackLeibler Distance all samples are grouped with different distances to the mean of these case, so Euclidean Distance and Kullback-Leibler Distance discrimines all the classes.

\subsection{Classification Examples}

Figure 15, shows the pattern classification obtained applying the distance Histogram to Centroid to a data base of 28 classes with 20 pattern for each class. The 


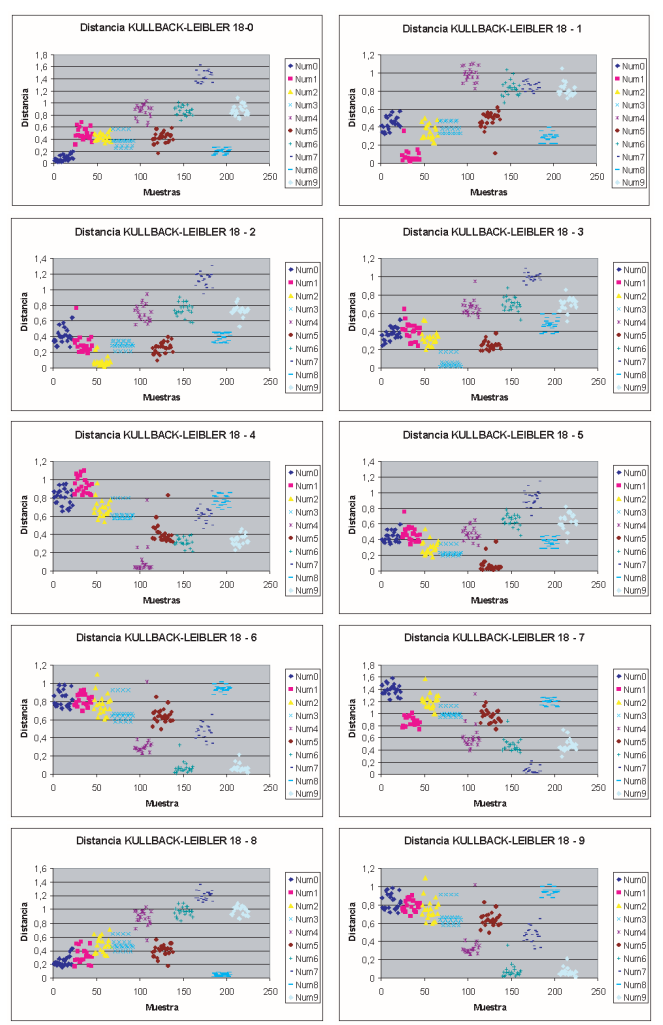

Figure 14: Kullback-Leibler Distance.

procedure for classification is k-nearest neighbour, the original image are in figure 10. A different colour is assigned to each class (figure 15 up) and the classification obtained is in figure 15 bottom.

As we can see, the digit 1 and the letter I are correctly recognized, and digit 6 and digit 9 have the same shape. Several proves to verify the distance histogram to Centroid have been made. The other digits and letters are correctly recognized.

\section{CONCLUSION}

A unique feature to recognize objects is defined. The main advantages are the elimination of the feature selection process and avoiding the problem of dimensionality.

The proposed system has been proved to be robust to discriminate between classes in a given set of objects. It could also be applied to other kind of objects when a fast classification is needed.

As a future work, we propose studying the adequate number of categories for each database case. We also plan to design a new suitable classification method to work in real time.
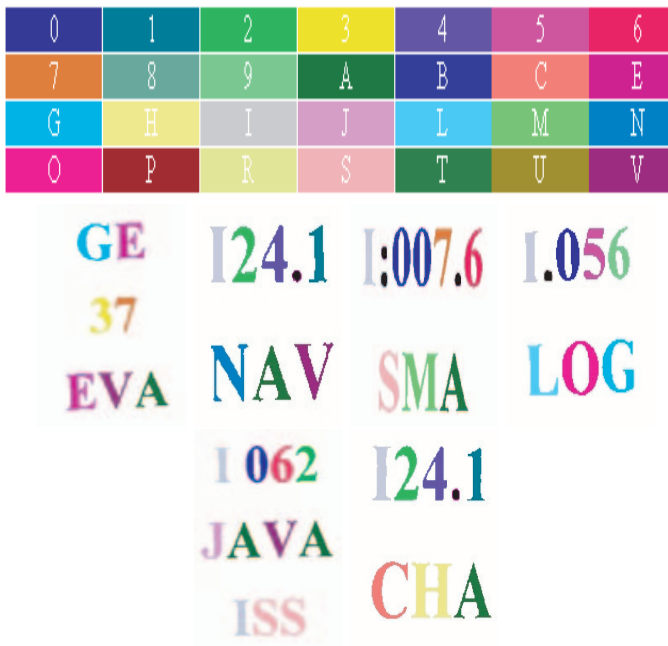

Figure 15: Recognized objects.

\section{REFERENCES}

Arques, P., Aznar, F., Pujol, M., and Rizo, R. (2005). Segmentacin de imgenes en tiempo real mediante umbralizacin adaptativa. In In 11th Conference of the Spanish Association for Artificial Intelligence.

Duda, R., Hart, P., and Strok, D. (2001). Pattern Classification. John Wiley and sons, New York, 2nd edition.

Fontoura, L. D. and Marcondes, R. (2001). Shape Analysis and Classification. Theory and Practice. CRC Press.

Marr, D. (1982). Vision. W.H. Freeman and Company.

Papathomas, T. (1996). Special issue on visual-perception: Guest editorial. In In International Journal of Imaging System and Technology.

Pitas, I. (2000). Digital Image Procession. Algorithms and Applications. John Wiley and sons, New York, 1st edition.

Theodoridis, S. and Koutroumbas, K. (1999). Pattern Recognition. Academic Press, London. 